\title{
A mathematical model for swallowing of concentrated fluids in oesophagus
}

\author{
S. K. Pandey and Dharmendra Tripathi* \\ Department of Applied Mathematics, Institute of Technology, Banaras Hindu University, Varanasi, India
}

\begin{abstract}
This model investigates particularly the impact of an integral and a non-integral number of waves on the swallowing of food stuff such as jelly, tomato puree, soup, concentrated fruits juices and honey transported peristaltically through the oesophagus. The fluid is considered as a Casson fluid. Emphasis is on the study of the dependence of local pressure distribution on space and time. Mechanical efficiency, reflux limit and trapping are also discussed. The effect of Casson fluid vis-à-vis Newtonian fluid is investigated analytically and numerically too. The result is physically interpreted as that the oesophagus makes more efforts to swallow fluids with higher concentration. It is observed that the pressure is uniformly distributed when an integral number of waves is there in the oesophagus; but it is non-uniform when a non-integral number of waves is present therein. It is further observed that as the plug flow region widens, the pressure difference increases, which indicates that the time-averaged flow rate will reduce for a Casson fluid. It is also concluded that Casson fluids are more prone to reflux.
\end{abstract}

Keywords: Peristalsis, oesophagus, casson fluid, mechanical efficiency, reflux, trapping

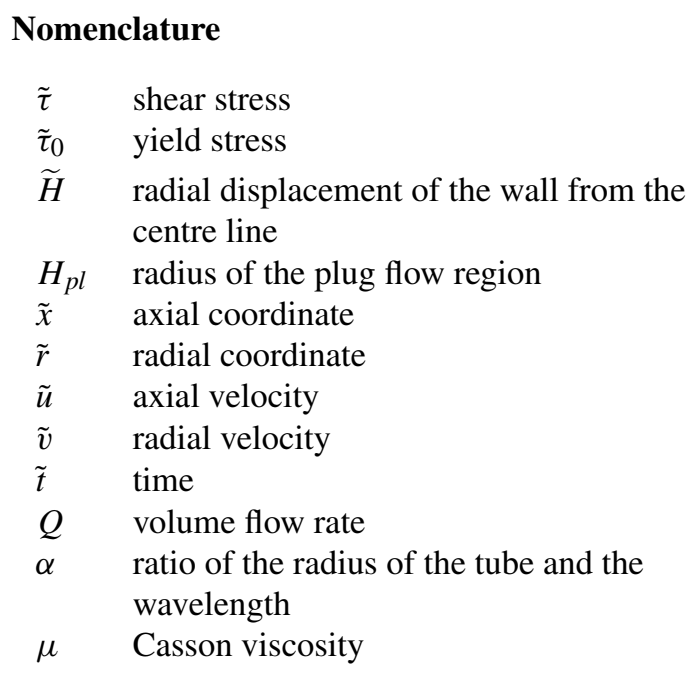

${ }^{*}$ Corresponding author: Dharmendra Tripathi, Department of Applied Mathematics, Institute of Technology, Banaras Hindu University, Varanasi-221005, India. E-mail: dtripathi.rs.apm@itbhu. ac.in.

$\begin{array}{ll}\dot{\gamma} & \text { rate of shear strain } \\ \tilde{\phi} & \text { amplitude } \\ l & \text { length of the tube } \\ c & \text { wave velocity } \\ \rho & \text { fluid density } \\ \tilde{p} & \text { pressure } \\ \lambda & \text { wavelength } \\ a & \text { radius of the tube } \\ \psi & \text { stream function } \\ \operatorname{Re} & \text { Reynolds number }\end{array}$

\section{Introduction}

Swallowing in the oesophagus is peristaltic in nature. Peristalsis requires no piston to pump the fluid contained in the duct. This simplifies pumping and ultimately saves the propellant from any sort of contamination that may hamper its purity. The mechanism involves involuntary periodic contraction of the wall followed by relaxation in the oesophagus. This leads 
to the rise of pressure gradient that eventually pushes the content forward. An interesting fact is that the cattle grazing in the fields have their necks down; yet they swallow without any difficulty. This illustrates that there is almost no role played by the gravity in swallowing through the oesophagus. The flow of urine in ureters, the vasomotion of blood, etc. are a few more physiological instances where peristalsis is dominant. We intend to investigate swallowing of semi-fluids such as jelly, tomato puree, honey, soup, and concentrated fruits juices etc. through oesophagus. The model assumed is the flow of a Casson fluid in a finite long circular cylindrical tube. The justification is given in the forthcoming paragraphs.

The human oesophagus is a flexible muscular tube extending from pharynx to stomach. Its length varies from $9 \mathrm{~cm}$ (for children) to $30 \mathrm{~cm}$ (for an adult) and the diameter lies between $1 \mathrm{~cm}$ and $2 \mathrm{~cm}$. Thus, the length and diameter ratio varies from $9: 1$ to $15: 1$. This gives a clear indication that the oesophageal length is finite in comparison to its diameter. However, most of the studies dealing with peristaltic transport consider infinite length tubes. Consequently, those results cannot be directly applied to the flows through the oesophagus. Taking into consideration the aforementioned facts, we attempt to model oesophagus as a circular cylindrical tube of finite length.

Recently, researchers investigated peristaltic transport of fluids modelled differently such as Casson fluid model [1-3], Maxwell fluid model [4, 5], Burgers' fluids [6], power-law fluids [7-9], particle-fluid mixture [10], micro-polar fluid [11-13], Herschel- Bulkley fluid [14, 15]. Misra and Pandey [1] modelled twolayered peristaltic flow of Casson fluid in an infinite channel and a tube and applied the model for blood flow. Mernone and Mazumadar [2] compared Newtonian and non-Newtonian Casson flows in an infinite channel by employing perturbation technique. Rani and Sarojamma [3] considered Casson flow in an asymmetric infinitely long channel and discussed the phenomena trapping and reflux. The rest cited above have dealt with some other non-Newtonian fluids considering infinite length geometry of the vessel.

Only a few investigations pertaining to peristalsis have considered finite length tubes. Li and Brasseur [16] investigated peristaltic transport of Newtonian fluid in a finite length tube and Misra and Pandey [17] extended the work for a power-law fluid. Li and Brasseur [16] further raised an issue of prime concern that the oesophageal wall undergoes contraction followed by relaxation; but in most of the studies the wall is considered to oscillate about the mean position, i.e., the stationary boundary. They incorporated this correction so that the model can be applied to oesophageal swallowing. It was further improved by a more suitable model by Misra and Pandey [17].

The masticated food-bolus can have diverse physical properties. The contents of the oesophagus are of various kinds from liquid to solid from physical point of view and from Newtonian to non-Newtonian from mechanical point of view. Recently, Pandey and Tripathi $[18,19]$ have reported their investigations on the flow properties of Maxwell and MHD fluids. Here, we aim to study the transportation of semi-fluids such as jelly, tomato puree, honey, soup, and concentrated fruits juices etc that are highly concentrated and resemble Casson fluid in nature (cf. [20, 21]).

Another important aspect of peristalsis in a finite length tube is that one can study non-integral number of waves in the train propagating along the wall of the tube. Li and Brasseur [16] put forward some important results in this regard for Newtonian fluids that we shall discuss wherever required. We try to investigate here those properties with regard to Casson fluids.

Shapiro et al. [22] discovered some inherent phenomena such as reflux and trapping and also deduced the mechanical efficiency of pumping. Without these an investigation appears incomplete. We intend to discuss reflux and mechanical efficiency in this paper.

\section{Mathematical formulation}

We consider oesophagus as a circular cylindrical tube of finite length. Since the masticated food is assumed to be Casson fluid, its constitutive equations may be given by

$$
\begin{array}{ll}
\sqrt{\tilde{\tau}}=\sqrt{\mu \dot{\gamma}}+\sqrt{\tilde{\tau}_{0}} & \text { for } \tilde{\tau} \geq \tilde{\tau}_{0}, \\
\dot{\gamma}=0 & \text { for } \tilde{\tau} \leq \tilde{\tau}_{0},
\end{array}
$$

where $\tilde{\tau}, \mu, \dot{\gamma}, \tilde{\tau}_{0}$ represent respectively shear stress, Casson viscosity, the rate of shear strain and yield stress.

The oesophagus undergoes a periodic contraction followed by successive relaxation (cf. Fig. 1). Therefore, the peristaltic radial wall motion of the tube is suitably modelled by the equation

$$
\tilde{H}(\tilde{x}, \tilde{t})=a-\tilde{\phi} \cos ^{2} \frac{\pi}{\lambda}(\tilde{x}-c \tilde{t}),
$$




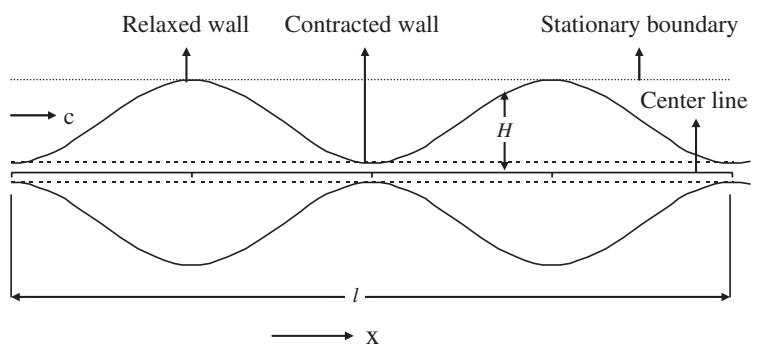

Fig. 1. The diagram, based on Eq. (10), represents the propagation of a progressive transverse wave along the walls of the tube containing fluid, which undergoes contraction and relaxation but no expansion beyond the boundary.

where $\tilde{H}, \tilde{x}, \tilde{t} a, \tilde{\phi}, \lambda$ and $c$ respectively denote radial displacement of the wall from the centre line, axial coordinate, time parameter, radius of the tube, amplitude of the wave supposed to propagate along the wall to create a motion as desired, wavelength, wave velocity, as suggested by Misra and Pandey [17].

\subsection{Analysis}

The governing equations for the axi-symmetric flow of an incompressible fluid are given by

$$
\begin{aligned}
& \rho \frac{D \tilde{u}}{D \tilde{t}}=-\frac{\partial \tilde{p}}{\partial \tilde{x}}+\tilde{\nabla} \cdot \tilde{\tau} \\
& \rho \frac{D \tilde{v}}{D \tilde{t}}=-\frac{\partial \tilde{p}}{\partial \tilde{r}}+\tilde{\nabla} \cdot \tilde{\tau}
\end{aligned}
$$

where $\frac{D}{D \tilde{t}} \cong \frac{\partial}{\partial \tilde{t}}+\tilde{u} \frac{\partial}{\partial \tilde{x}}+\tilde{v} \frac{\partial}{\partial \tilde{r}}, \tilde{\nabla} \equiv \frac{\partial}{\partial \tilde{x}}+\frac{1}{\tilde{r}} \frac{\partial}{\partial \tilde{r}}$, and the continuity equation is

$$
\frac{\partial \tilde{u}}{\partial \tilde{x}}+\frac{1}{\tilde{r}} \frac{\partial(\tilde{r} \tilde{v})}{\partial \tilde{r}}=0,
$$

where $\rho, \tilde{u}, \tilde{p}, \tilde{v}$ and $\tilde{r}$ are fluid density, axial velocity, pressure, radial velocity and radial coordinate.

The various parameters are non-dimensionalised as follows:

$$
\left.\begin{array}{c}
x=\frac{\tilde{x}}{\lambda}, r=\frac{\tilde{r}}{a}, t=\frac{c \tilde{t}}{\lambda}, u=\frac{\tilde{u}}{c}, v=\frac{\tilde{v}}{c \alpha}, \\
\alpha=\frac{a}{\lambda}, H=\frac{\tilde{H}}{a}, \tau=\frac{\tilde{\tau} a}{\mu c}, \\
\tau_{0}=\frac{\tilde{\tau}_{0} a}{\mu c}, l=\frac{\tilde{l}}{\lambda}, \phi=\frac{\tilde{\phi}}{a}, p=\frac{\tilde{p} a^{2}}{\mu c \lambda}, \\
\psi=\frac{\tilde{\psi}}{\pi a^{2} c}, Q=\frac{\tilde{Q}}{\pi a^{2} c}, \operatorname{Re}=\frac{\rho c a \alpha}{\mu},
\end{array}\right\}
$$

where $\alpha$ is a parameter that gives the ratio of the radius of the tube and the wavelength, while $l, \psi, Q$ and $\operatorname{Re}$ are respectively length of the tube, stream function, volume flow rate and Reynolds number.

Under the long wavelength approximation and consequently, under the low Reynolds number approximation, Eqs. (1-6) reduce respectively to the following dimensionless form:

$$
\begin{aligned}
& \sqrt{\tau}=\sqrt{\tau_{0}}+\sqrt{\frac{\partial u}{\partial r}} \quad \text { for } \tau \geq \tau_{0}, \\
& \frac{\partial u}{\partial r}=0 \quad \text { for } \tau \leq \tau_{0}, \\
& H(x, t)=1-\phi \cos ^{2} \pi(x-t), \\
& \frac{\partial p}{\partial x}=\frac{1}{r} \frac{\partial(r \tau)}{\partial r}, \\
& \frac{\partial p}{\partial r}=0, \\
& \frac{\partial u}{\partial x}+\frac{1}{r} \frac{\partial(r v)}{\partial r}=0 .
\end{aligned}
$$

The following boundary conditions, given in the dimensionless form (dimensional counterparts being similar), are imposed on the governing equations:

no slip condition on inner surface of the

$$
\text { oesophagus, i.e., }\left.u(x, r, t)\right|_{r=H}=0 \text {, }
$$

radial velocity at the wall of the oesophagus,

$$
\text { i.e., }\left.v(x, r, t)\right|_{r=H}=\frac{\partial H}{\partial t},
$$

absence of any radial velocity in the plug

$$
\begin{gathered}
\text { flow region, i.e., }\left.v(x, r, t)\right|_{r=H_{p l}}=0, \\
\text { regularity condition, i.e., }\left.\frac{\partial u}{\partial r}(x, r, t)\right|_{r=H_{p l}}=0,
\end{gathered}
$$

where $H_{p l}$ is the radius of the plug flow region, and it is defined by

$$
H_{p l}=2 \tau_{0} / \frac{\partial p}{\partial x} .
$$

\subsection{Solution}

Integrating Eq. (11) once with respect to $r$, we get

$$
\tau=\frac{A}{r}+\frac{r}{2} \frac{\partial p}{\partial x}
$$

where $A$ is an arbitrary function of $x$ and $t$. 
Using Eq. (19) into Eq. (8) and applying the boundary condition (17), we obtain

$$
\frac{\partial u}{\partial r}=\frac{1}{2} \frac{\partial p}{\partial r}\left\{r+H_{p l}-2 \sqrt{r H_{p l}}\right\} .
$$

Further, integration of Eq. (20) once again from $r$ to $H$ followed by application of the no-slip condition (14) yields

$$
\begin{aligned}
u= & \frac{1}{4} \frac{\partial p}{\partial x}\left\{(r-H)\left(r+H+2 H_{p l}\right)\right. \\
& \left.-\frac{8}{3} \sqrt{H_{p l}}\left(r^{\frac{3}{2}}-H^{\frac{3}{2}}\right)\right\} .
\end{aligned}
$$

The plug flow velocity is deduced from Eq. (21) by the substitution $r=H_{p l}$ as

$$
\begin{aligned}
u_{p l}= & \frac{1}{4} \frac{\partial p}{\partial x}\left\{\left(H_{p l}-H\right)\left(H+3 H_{p l}\right)\right. \\
& \left.-\frac{8}{3} \sqrt{H_{p l}}\left(H_{p l}^{\frac{3}{2}}-H^{\frac{3}{2}}\right)\right\} .
\end{aligned}
$$

Solving the continuity equation (13), by applying Eq. (21) together with the boundary condition (16), the radial velocity is given by

$$
\begin{aligned}
v & =\frac{1}{2}\left[\frac{1}{2 r} \frac{\partial p}{\partial x} \frac{\partial H}{\partial x}\left(r^{2}-H_{p l}^{2}\right)\left(H+H_{p l}-2 \sqrt{H H_{p l}}\right)\right. \\
- & \frac{\partial^{2} p}{\partial x^{2}}\left\{\frac{r}{8}\left(r^{2}-2 H^{2}\right)+\frac{r H_{p l}}{6}(2 r-3 H)\right. \\
& -\frac{8}{21} r \sqrt{H_{p l}}\left(r^{\frac{3}{2}}-\frac{7}{4} H^{\frac{3}{2}}\right)+\frac{H_{p l}^{2}}{r} \\
& \left.\left.\times\left(H^{2}+2 H H_{p l}-\frac{13}{168} H_{p l}^{2}-\frac{8}{3} H \sqrt{H H_{p l}}\right)\right\}\right] .
\end{aligned}
$$

By employing the boundary condition (15), the radial velocity at the wall yields

$$
\begin{aligned}
& H \frac{\partial H}{\partial t}=\frac{1}{4} \frac{\partial p}{\partial x} \frac{\partial H}{\partial x}\left(H^{2}-H_{p l}^{2}\right)\left(H+H_{p l}-2 \sqrt{H H_{p l}}\right) \\
& +\frac{1}{2} \frac{\partial^{2} p}{\partial x^{2}}\left\{H^{3}\left(\frac{H}{8}+\frac{H_{p l}}{6}-\frac{2}{7} \sqrt{H H_{p l}}\right)\right. \\
& \left.-H_{p l}^{2}\left(\frac{H^{2}}{4}+\frac{H H_{p l}}{2}-\frac{2 H}{3} \sqrt{H H_{p l}}-\frac{13}{168} H_{p l}^{2}\right)\right\} .
\end{aligned}
$$

The pressure gradient is then derived from Eq. (24) and is given by

$$
\begin{gathered}
\frac{\partial p}{\partial x}=\frac{G(t)+\int_{0}^{x} H \frac{\partial H}{\partial t} d s}{\frac{H^{3}}{48}\left(3 H+4 H_{p l}\right)-\frac{H_{p l}^{2}}{336}\left(42 H^{2}\right.} \\
\left.-13 H_{p l}^{2}+84 H H_{p l}\right) \\
-\frac{H \sqrt{H H_{p l}}}{21}\left(3 H^{2}-7 H_{p l}^{2}\right)
\end{gathered}
$$

where $G(t)$ is the arbitrary function of $t$.

The pressure at an arbitrary point along the length of the oesophagus is determined by integrating Eq. (25) from the inlet to the arbitrary axial point, which gives

$$
\begin{aligned}
& p(x, t)-p(0, t)=\int_{0}^{x} \frac{G(t)+\int_{0}^{s} H \frac{\partial H}{\partial t} d s_{1}}{\frac{H^{3}}{48}\left(3 H+4 H_{p l}\right)-\frac{H_{p l}^{2}}{336}} d s, \\
& \left(42 H^{2}-13 H_{p l}^{2}+84 H H_{p l}\right) \\
& -\frac{H \sqrt{H H_{p l}}}{21}\left(3 H^{2}-7 H_{p l}^{2}\right)
\end{aligned}
$$

so that the pressure difference between the two ends of the oesophagus is

$$
\begin{aligned}
p(l, t)-p(0, t)= & \int_{0}^{l} \frac{G(t)+\int_{0}^{x} H \frac{\partial H}{\partial t} d s}{H^{3}}\left(3 H+4 H_{p l}\right)-\frac{H_{p l}^{2}}{336} \\
& \left(42 H^{2}-13 H_{p l}^{2}+84 H H_{p l}\right) \\
& -\frac{H \sqrt{H H_{p l}}}{21}\left(3 H^{2}-7 H_{p l}^{2}\right)
\end{aligned}
$$

from which $G(t)$ can be obtained as 


$$
G(t)=\frac{p(l, t)-p(0, t)-\int_{0}^{l} \frac{\int_{0}^{x} H \frac{\partial H}{\partial t} d s}{\frac{H^{3}}{48}\left(3 H+4 H_{p l}\right)-\frac{H_{p l}^{2}}{336}\left(42 H^{2}-13 H_{p l}^{2}+84 H H_{p l}\right)-\frac{H \sqrt{H H_{p l}}}{21}\left(3 H^{2}-7 H_{p l}^{2}\right)} d x}{\int_{0}^{l} \frac{1}{\frac{H^{3}}{48}\left(3 H+4 H_{p l}\right)-\frac{H_{p l}^{2}}{336}\left(42 H^{2}-13 H_{p l}^{2}+84 H H_{p l}\right)-\frac{H \sqrt{H H_{p l}}}{21}\left(3 H^{2}-7 H_{p l}^{2}\right)} d x} .
$$

The volume flow rate for the wave-train is defined as $Q(x, t)=2 \int_{H_{p l}}^{H} u r d r$, which, in conjunction with Eq. (21), yields

$$
\begin{aligned}
Q(x, t)= & -\frac{\partial p}{\partial x}\left\{\frac{H^{3}}{24}\left(3 H+4 H_{p l}\right)\right. \\
& -\frac{H_{p l}^{2}}{168}\left(42 H^{2}-13 H_{p l}^{2}+84 H H_{p l}\right) \\
& \left.-\frac{2 H \sqrt{H H_{p l}}}{21}\left(3 H^{2}-7 H_{p l}^{2}\right)\right\} .
\end{aligned}
$$

For $H_{p l}=0$, Eqs. (25-29) reduce to the corresponding equations derived by $\mathrm{Li}$ and Brasseur [16] for Newtonian fluids.

Averaging the volume flow rate for one time period, the time-averaged volume flow rate is found to be

$$
\begin{aligned}
\bar{Q}(x)= & \int_{0}^{1}-\frac{\partial p}{\partial x}\left\{\frac{H^{3}}{24}\left(3 H+4 H_{p l}\right)\right. \\
& -\frac{H_{p l}^{2}}{168}\left(42 H^{2}-13 H_{p l}^{2}+84 H H_{p l}\right) \\
& \left.-\frac{2 H \sqrt{H H_{p l}}}{21}\left(3 H^{2}-7 H_{p l}^{2}\right)\right\} d t,
\end{aligned}
$$

which, using the transformation between laboratory frame and wave frame, given later in Eq. (40), is expressed in terms of the flow rate in the wave and the laboratory frames respectively as

$\bar{Q}=q+1-\phi+\frac{3 \phi^{2}}{8}=Q-H^{2}+1-\phi+\frac{3 \phi^{2}}{8}$, so that the pressure gradient can be expressed from Eq. (29) as

$$
\begin{aligned}
\frac{\partial p}{\partial x}=- & \frac{\bar{Q}+H^{2}-1+\phi-\frac{3 \phi^{2}}{8}}{\frac{H^{3}}{24}\left(3 H+4 H_{p l}\right)-\frac{H_{p l}^{2}}{168}\left(42 H^{2}-13 H_{p l}^{2}\right.} \\
& \left.+84 H H_{p l}\right)-\frac{2 H \sqrt{H H_{p l}}}{21}\left(3 H^{2}-7 H_{p l}^{2}\right)
\end{aligned} .
$$

Integrating $\partial p / \partial x$ from 0 to $x$, the pressure difference between the inlet and an arbitrary point on the axis may be given by

$$
\begin{aligned}
p(x)-p(0)=-\int_{0}^{x} \frac{\bar{Q}+H^{2}-1+\phi-\frac{3 \phi^{2}}{8}}{\frac{H^{3}}{24}\left(3 H+4 H_{p l}\right)-\frac{H_{p l}^{2}}{168}} d s . \\
\\
\left.\quad 42 H^{2}-13 H_{p l}^{2}+84 H H_{p l}\right) \\
\quad-\frac{2 H \sqrt{H H_{p l}}}{21}\left(3 H^{2}-7 H_{p l}^{2}\right)
\end{aligned}
$$

Applying Eq. (20), the local wall shear stress $\tau_{w}=\left(\frac{\partial u}{\partial r}\right)_{r=H}$, may be given as $\tau_{w}=\frac{1}{2}\left(H+H_{p l}\right.$ $\left.-2 \sqrt{H H_{p l}}\right) \frac{\partial p}{\partial x}$, which, in view of Eq. (25), further reduces to 


$$
\tau_{w}=\frac{1}{2} \frac{\left(H+H_{p l}-2 \sqrt{H H_{p l}}\right)\left\{G(t)+\int_{0}^{x} H \frac{\partial H}{\partial t} d s\right\}}{\frac{H^{3}}{48}\left(3 H+4 H_{p l}\right)-\frac{H_{p l}^{2}}{336}}+.
$$

For $H_{p l}=0$, Eq. (34) reduces to the corresponding equation derived by Li and Brasseur [16] for Newtonian fluids.

Moreover, the yield stress can be deduced from Eqs. (25) \& (18) as where $\Delta p_{1}$, i.e., $p(1)-p(0)$, is the pressure difference across one wavelength and is given by

$$
\begin{aligned}
\Delta p_{1}=-\int_{0}^{1} & \frac{\bar{Q}+H^{2}-1+\phi-\frac{3 \phi^{2}}{8}}{\frac{H^{3}}{24}\left(3 H+4 H_{p l}\right)-\frac{H_{p l}^{2}}{168}} d x, \\
& \left(42 H^{2}-13 H_{p l}^{2}+84 H H_{p l}\right) \\
& -\frac{2 H \sqrt{H H_{p l}}}{21}\left(3 H^{2}-7 H_{p l}^{2}\right)
\end{aligned}
$$

$I_{11}=\int_{0}^{1} \frac{\partial p}{\partial x} \cos (2 \pi x) d x, I_{12}=\int_{0}^{1} \frac{\partial p}{\partial x} \cos ^{4}(\pi x) d x$,

and the maximum average flow rate (i.e. average flow rate for $\Delta p_{1}=0$ ) is obtained as

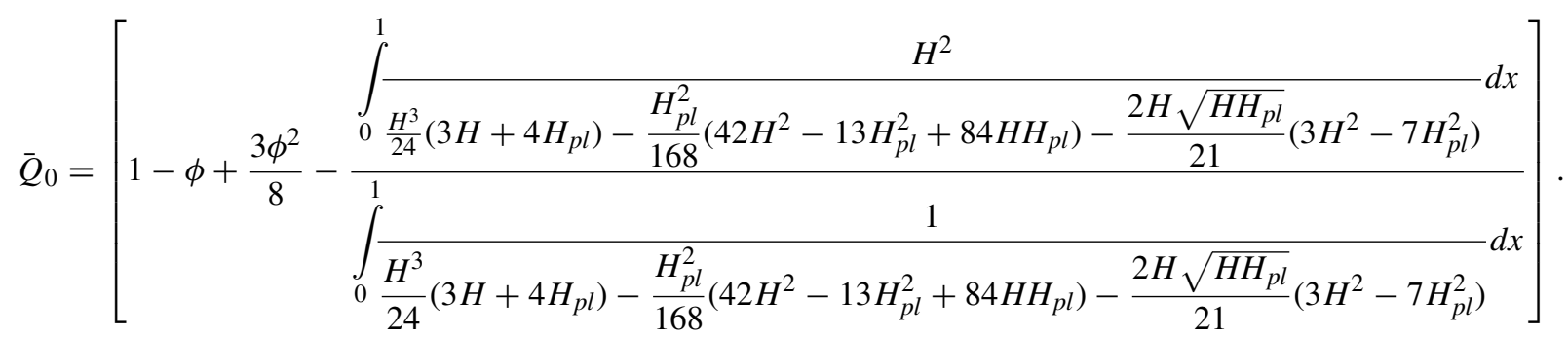

$$
\begin{gathered}
\tau_{0}=\frac{1}{2} \frac{H_{p l}\left\{G(t)+\int_{0}^{x} H \frac{\partial H}{\partial t} d s\right\}}{\frac{H^{3}}{48}\left(3 H+4 H_{p l}\right)-\frac{H_{p l}^{2}}{336}\left(42 H^{2}-13 H_{p l}^{2}\right.} . \\
\left.+84 H H_{p l}\right)-\frac{H \sqrt{H H_{p l}}}{21}\left(3 H^{2}-7 H_{p l}^{2}\right)
\end{gathered}
$$

\section{Mechanical efficiency}

Shapiro et al. [22] state that the mechanical efficiency of peristaltic pumping is the ratio of the rate, at which the useful energy (averaged over a period per wavelength) is stored in the fluid, to the mechanical work (averaged over a period per wavelength) delivered to the wall from outside agencies. The mechanical efficiency, in dimensionless form, of peristaltic pump during the transportation of Casson fluid, for the wall motion (13), is hence derived as

$$
E=\frac{\bar{Q} \Delta p_{1}}{2 \phi\left[I_{11}+\phi I_{12}-(1+\phi) \Delta p_{1}\right]},
$$

\section{Reflux limit}

Reflux is a phenomenon inherent to peristalsis (Shapiro et al. [22]). It refers to the retrograde motion near the wall. Analysis related to the estimation of reflux limit is as follows:

Dimensional form of stream function in the wave frame is defined as

$$
d \tilde{\psi}=2 \pi \tilde{R}(\tilde{U} d \tilde{R}-\tilde{V} d \tilde{X}),
$$

where $\tilde{\psi}, \tilde{U}, \tilde{X}, \tilde{V}$ and $\tilde{R}$ are the stream function, the axial velocity, the axial coordinate, the radial velocity and the radial coordinate respectively. Applying the following transformation between the wave frame and the laboratory frame, given by

$$
\left.\begin{array}{l}
X=x-t, R=r, U=u-1, \\
V=v, q=Q-r^{2}, \Psi=\psi-r^{2}
\end{array}\right\},
$$

where the parameters on the left side are in the wave frame and those on the right side are in the laboratory frame. Using Eq. (39) and the transformations (40), the stream function, in the dimensionless form, is derived as 
$\psi=-\frac{1}{2}\left[\frac{\left(\bar{Q}+H^{2}-1+\phi-\frac{3 \phi^{2}}{8}\right)\left\{\frac{r^{4}}{4}-\frac{r^{2} H^{2}}{2}+2 H_{p l}\left(\frac{r^{3}}{3}-\frac{r^{2} H}{2}\right)-\frac{16}{21} \sqrt{H_{p l}}\left(r^{\frac{7}{2}}-\frac{7}{4} r^{2} H^{\frac{3}{2}}\right)\right\}}{\frac{H^{3}}{24}\left(3 H+4 H_{p l}\right)-\frac{H_{p l}^{2}}{168}\left(42 H^{2}-13 H_{p l}^{2}+84 H H_{p l}\right)-\frac{2 H \sqrt{H H_{p l}}}{21}\left(3 H^{2}-7 H_{p l}^{2}\right)}+r^{2}\right]$.

The stream function $\psi_{w}$ at the wall is

$$
\left.\psi\right|_{r=H}=\bar{Q}-1+\phi-\frac{3 \phi^{2}}{8} .
$$

The reflux flow rate $Q_{\psi}(x)$ is defined as

$$
Q_{\psi}(x)=\psi+r^{2}(\psi, x)
$$

We average the above equation for one cycle to obtain the averaged reflux flow rate

$$
\bar{Q}_{\psi}=\psi+\int_{0}^{1} r^{2}(\psi, x) d x .
$$

In order to evaluate above integration using the perturbation method, we expand $r^{2}(\psi, x)$ in a power series in terms of a small parameters $\varepsilon$ about the wall as

$$
r^{2}(\psi, x)=H^{2}+a_{1} \varepsilon+a_{2} \varepsilon^{2}+\cdots
$$

Solving Eqs. (41) \& (45) and comparing the coefficient of $\varepsilon, \varepsilon^{2} \ldots$ on the two sides, we get

$$
a_{1}=-1 \text {, }
$$

$$
a_{2}=-\frac{1}{8 H}\left[\frac{\left(\sqrt{H}-\sqrt{H_{p l}}\right)^{2}\left(\bar{Q}+H^{2}-1+\phi-\frac{3 \phi^{2}}{8}\right)}{\frac{H^{3}}{24}\left(3 H+4 H_{p l}\right)-\frac{H_{p l}^{2}}{168}\left(42 H^{2}-13 H_{p l}^{2}+84 H H_{p l}\right)-\frac{2 H \sqrt{H H_{p l}}}{21}\left(3 H^{2}-7 H_{p l}^{2}\right)}\right] .
$$

The reflux condition is $\frac{\bar{Q}_{\psi}}{\bar{Q}}>1$, as $\varepsilon \rightarrow 0$, which gives the reflux limit for the Casson fluid as

\section{Numerical results and discussion}

Since the mathematical model involves expressions that cannot be integrated by classical methods, we opted for numerical solution. We wrote computer programs in $\mathrm{C}$ and used some numerical techniques to evaluate the integrals.

Once the masticated food, rolled into a bolus, enters into the oesophagus, the upper sphincter is blocked, averting any possibility of retrograde motion. The lower sphincter too remains closed until a food bolus arrives at and knocks it. Similar patterns of pressure distribution were found in experimental observations reported by Dodds [23] and Ren et al. [24].

\subsection{Integral number of waves}

In order to study the local dynamics, we consider a train of two waves propagating along the wall of the

$$
\bar{Q}<1-\phi+\frac{3 \phi^{2}}{8}-\frac{\int_{0}^{1} \frac{H\left(\sqrt{H}-\sqrt{H_{p l}}\right)^{2}}{\frac{H^{3}}{24}\left(3 H+4 H_{p l}\right)-\frac{H_{p l}^{2}}{168}\left(42 H^{2}-13 H_{p l}^{2}+84 H H_{p l}\right)-\frac{2 H \sqrt{H H_{p l}}}{21}\left(3 H^{2}-7 H_{p l}^{2}\right)} d x}{\int_{0}^{1} \frac{\frac{1}{H}\left(\sqrt{H}-\sqrt{H_{p l}}\right)^{2}}{\frac{H^{3}}{24}\left(3 H+4 H_{p l}\right)-\frac{H_{p l}^{2}}{168}\left(42 H^{2}-13 H_{p l}^{2}+84 H H_{p l}\right)-\frac{2 H \sqrt{H H_{p l}}}{21}\left(3 H^{2}-7 H_{p l}^{2}\right)} d x} .
$$

oesophagus which contains a Casson fluid to be swallowed. Initially, we investigate the temporal effects as well as the influence of yield stress on the pressure 
distribution between the two ends of the oesophagus, which are kept identically at zero pressure, i.e., $p(l, t)=p(0, t)=0$. This is termed as free pumping.

We aim to study the effect of the plug flow region on the general pumping performance. It is observed that the pressure distributions at the instants $t=0.0-1.0$ with intervals 0.25 (cf. Fig. 2) reveal qualitative similarity with the flows of Newtonian and also the power-law fluids (cf. [17]).

For a fixed plug flow region determined by $H_{p l}$, it is observed that as soon as a bolus steps into the oesophagus at $t=0$ (cf. Fig. 2a), the pressure at the inlet rises near the tail of the bolus and it is sufficient to restrain a possible retrograde motion. The pressure falls then to zero at the mid-point of the bolus, which further diminishes to its lower peak. Then it rises almost vertically to its upper peak near the head of the bolus, which is equal to the previous maximum value. This rise of pressure can be attributed to the requirement that can prevent a flow in the opposite direction. The pressure distribution, for the leading bolus is identical. It is further noticed that the final pressure at the head of the leading bolus is zero as expected. This rise of pressure to zero indicates a controlled motion of the content which is ready to be delivered to the stomach through the cardiac sphincter which permanently sieges any possible retrograde motion by closing the sphincter.

When $t=0.25$ (cf. Fig. 2b), one fourth of a new bolus has undergone swallowing process into the oesophagus and one fourth of the leading bolus has entered into the stomach. The pressure at the inlet starts waning to help the incoming bolus enter and the pressure declining from the peak to zero at the outlet favours the movement into the stomach.

For $t=0.5$ and $t=0.75$, depicted by Fig. $2 \mathrm{c}, \mathrm{d}$ respectively, the pressure distributions unfold a continuous process of fluid movement in the oesophagus. When $t=1.0$, the pressure distribution exhibits total

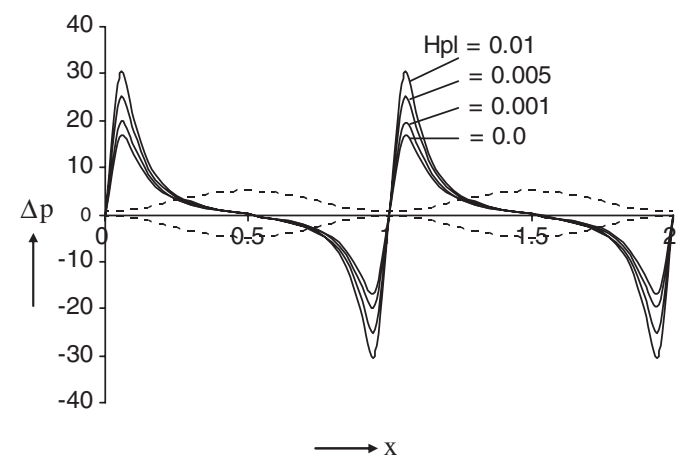

(a) $t=0.0$ (and also for $t=1.0$ )

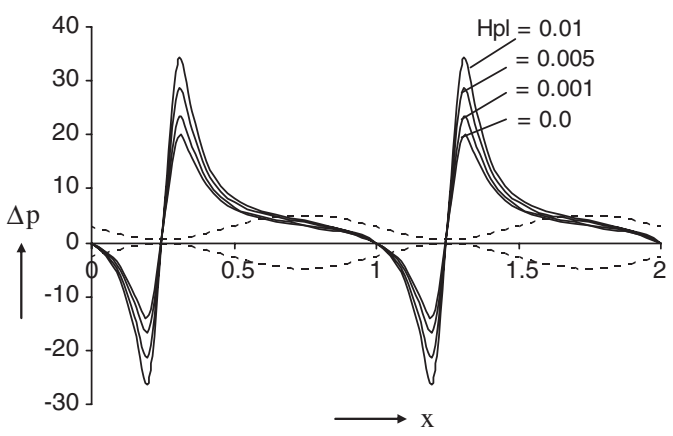

(b) $t=0.25$

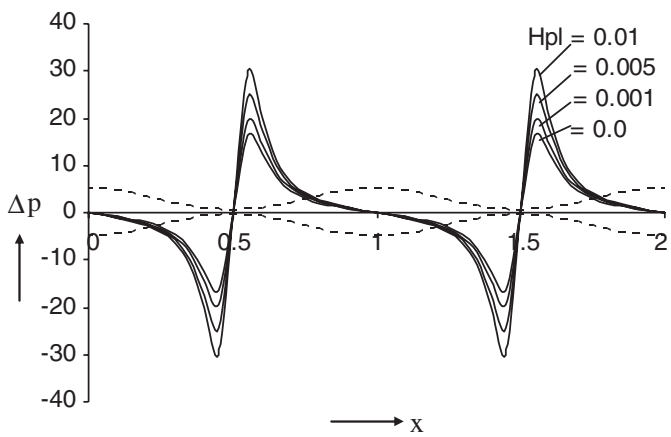

(c) $t=0.5$

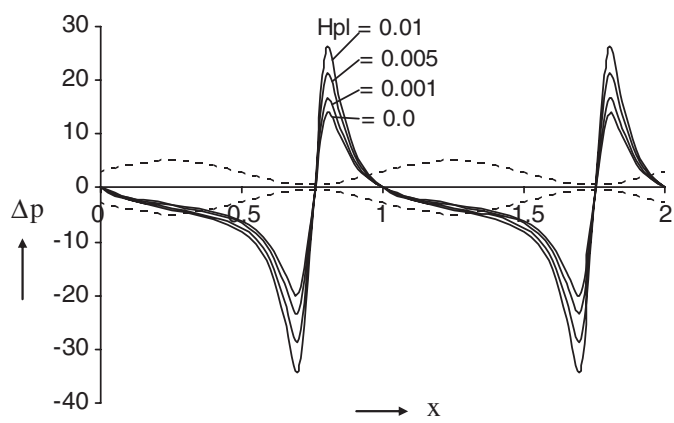

(d) $t=0.75$

Fig. 2. Pressure difference vs. axial distance. Dotted lines represent the position of bolus and solid lines show the pressure distribution, based on Eq. (26), across length of the tube passing Casson fluid for different radii of plug flow region $\left(H_{p l}=0.0,0.001,0.005,0.01\right), \phi=0.9$ and $l=2.0$. 
similarity with that at $t=0$. This indicates that the oesophagus is waiting to accept a new food bolus as the previous one has already been pushed into the stomach.

The magnitude of the pressure along the oesophagus increases with the thickness of the plug flow region. Increasing thickness of the plug flow region is an indication of enhancing non-Newtonian properties of the fluid. This is a revelation that the oesophagus has to be functionally more efficient to push the fluid forward. This inference is favoured by another revelation that we shall discuss later while discussing mechanical efficiency.

Local wall shear stress distribution along the oesophageal length shows further similarity with Newtonian fluid, i.e., $H_{p l}=0$ (cf. [17]). It is observed that $H_{p l}$, the only additional parameter that attributes to Casson nature of the fluid substantially increases local wall shear stress (cf. Fig. 3).

Figure 4, based on Eq. (35), reveal another interesting result that gives yield stress distribution along the oesophagus in the plug flow region. For a fixed $H_{p l}$, it is observed that the yield stress distribution is similar to local wall shear stress distribution. However, it is quantitatively quite small.

\subsection{Non-integral number of waves}

The foregoing analyses carried out for integral number of waves are also performed for the case of non-integral number of waves. This case is inherent to finite length tubes. We take $l=1.8$ to investigate the effects of non-integral number of waves.

In this case the pressure distributions at those instants (cf. Fig. 5) are observed to be dissimilar qualitatively as well as quantitatively from the previous case. Only the leftward pressure peaks are identical with those of the previous case for $t=0,1.0$; but the pressure distribution for the rightward wave which is partly accommodated in the length of the tube is different for higher values of the pressure peaks. The

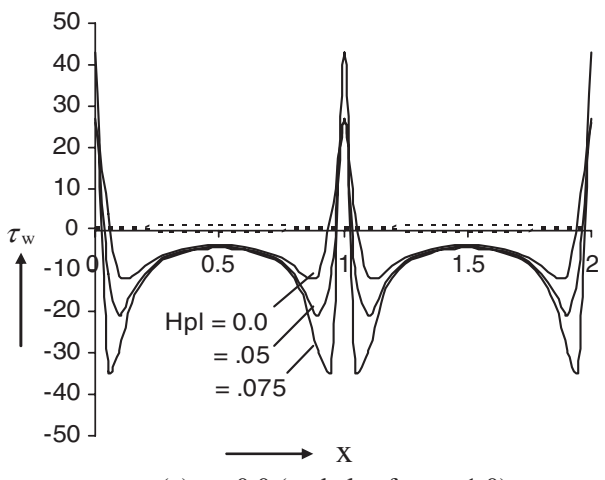

(a) $t=0.0$ (and also for $t=1.0$ )

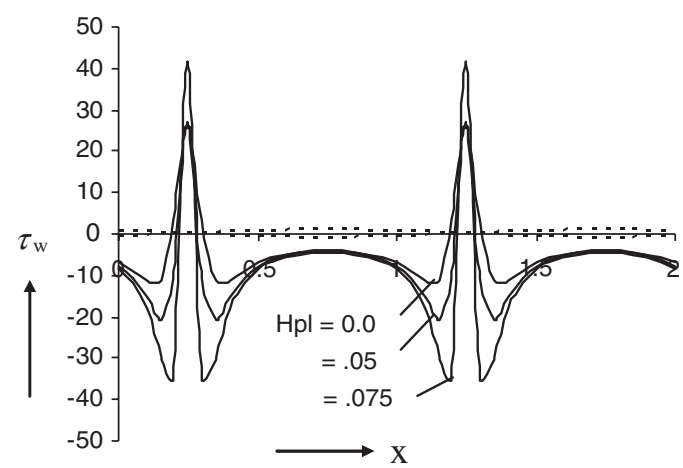

(b) $t=0.25$

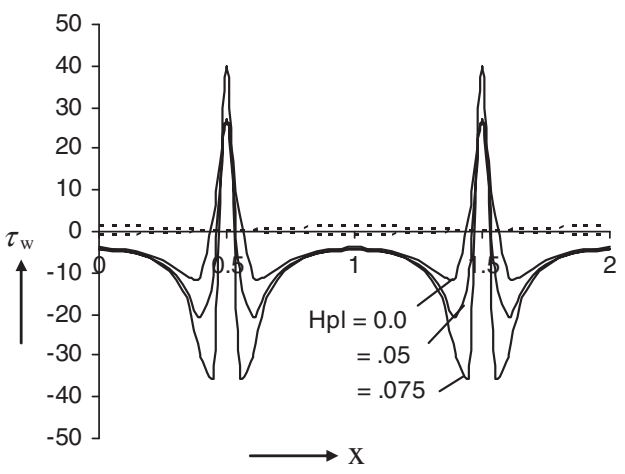

(c) $t=0.5$

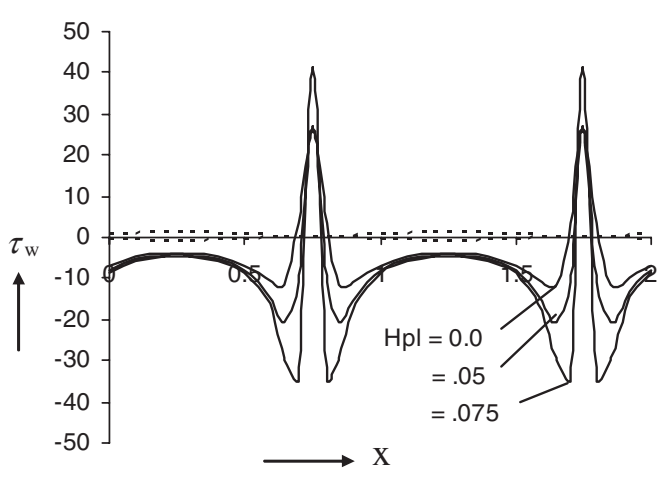

(d) $t=0.75$

Fig. 3. Local wall shear stress vs. axial distance along the tube. Dotted lines (...) represent the position of wave, whereas continuous lines (8) represent the local wall shear stress distribution, based on Eq. (34), for different radii of plug flow region $\left(H_{p l}=0.0,0.05,0.075\right), \phi=.9$ and $l=2.0$. 


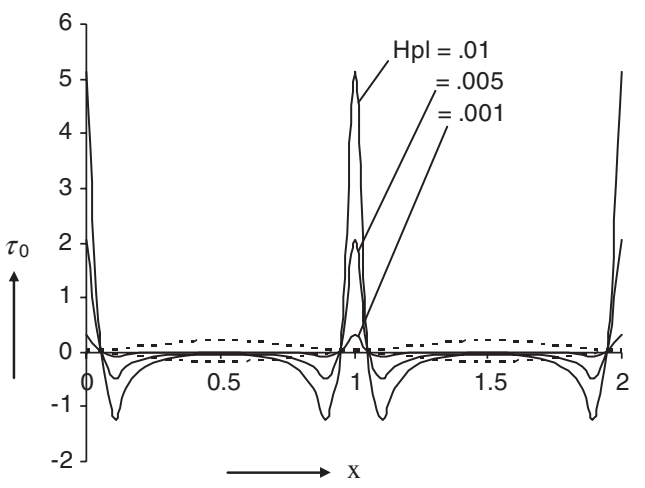

(a) $t=0.0$ (and also for $t=1.0$ )

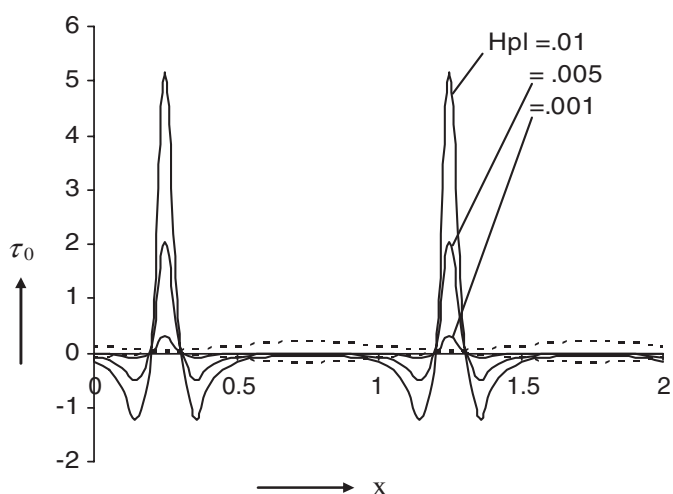

(b) $t=0.25$

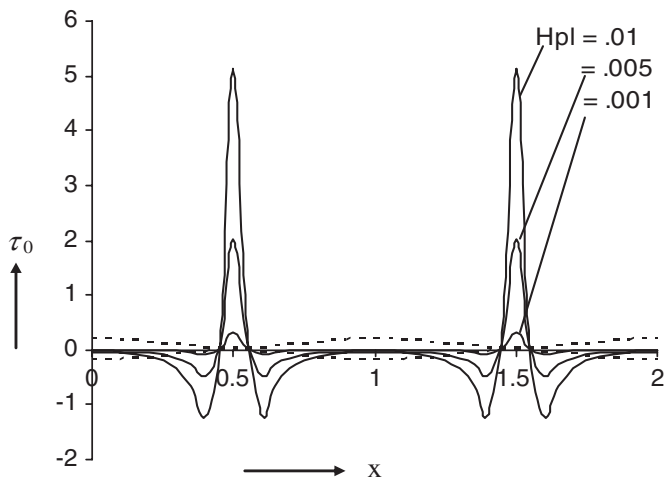

(c) $t=0.5$

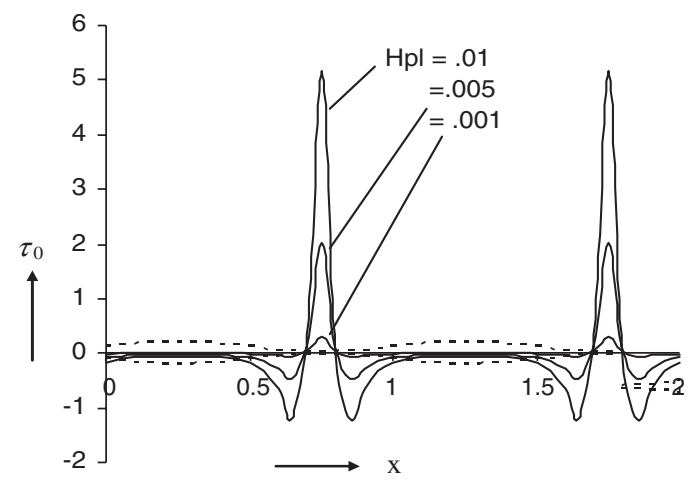

(d) $t=0.75$

Fig. 4. Yield stress vs. axial distance along the tube at five time instants. Dotted lines (....) represent the position of wave, whereas continuous lines (8) represent the yield stress distribution, based on Eq. (35), for different radii of plug flow region $\left(H_{p l}=0.001,0.005,0.01\right), \phi=.9$ and $l=2.0$.

pressure distribution looks complex with more uneven distribution. For the second bolus, the increased pressure diminishes to zero at the end without acquiring negative value for $t=0,1.0$ while it rises to only zero from negative values for $t=0.75$. The case $t=0.5$ is an exception. Similar complexity is foreseen for local wall shear stress. We therefore leave aside any further discussion in this regard.

\subsection{Mechanical efficiency}

Figure 6 is a graph, based on Eq. (36), plotted for mechanical efficiency vs. the ratio of the time-averaged flow rate to the maximum averaged flow rate. It reveals that the efficiency of an oesophagus carrying Casson fluid increases with the thickness of the plug flow region. This favours a conclusion discussed earlier in this section that the oesophagus is required to be more efficient to swallow a Casson fluid.

\subsection{Pressure difference across one wavelength vs. time-averaged flow rate}

Further, we consider the case where there is a difference of pressures at the two ends of the pump across one wavelength. Pressure difference required to refrain the flow completely is positive and maximum. It is observed that pressure difference across one wavelength of the oesophagus declines for a higher averaged flow rate for a fixed $H_{p l}$. As the plug flow region widens, the pressure difference increases for a fixed time-averaged flow rate (cf. Fig. 7). The graph further reveals that the maximum time-averaged flow rate is dependent on the thickness of the plug flow region.

\subsection{Reflux}

Figure 8 depicts the impact of the plug flow region on the reflux limit. It is observed that the reflux region 


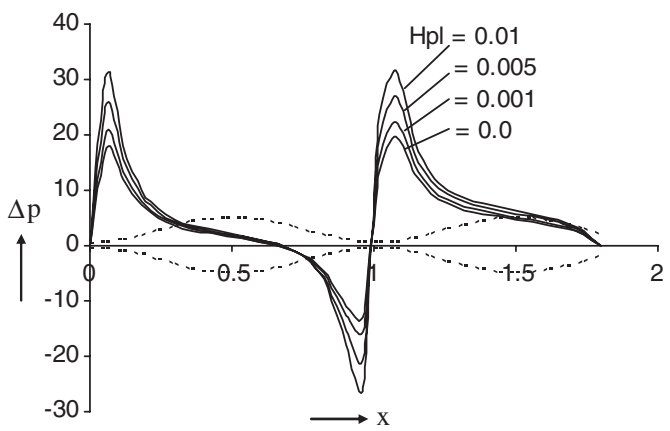

(a) $t=0.0$ (and also for $t=1.0$ )

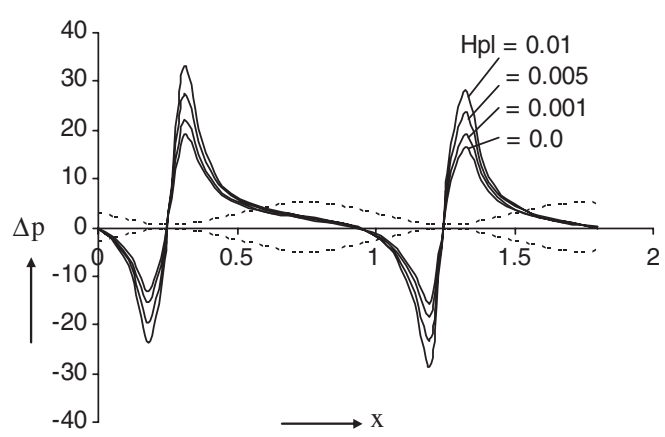

(b) $t=0.25$

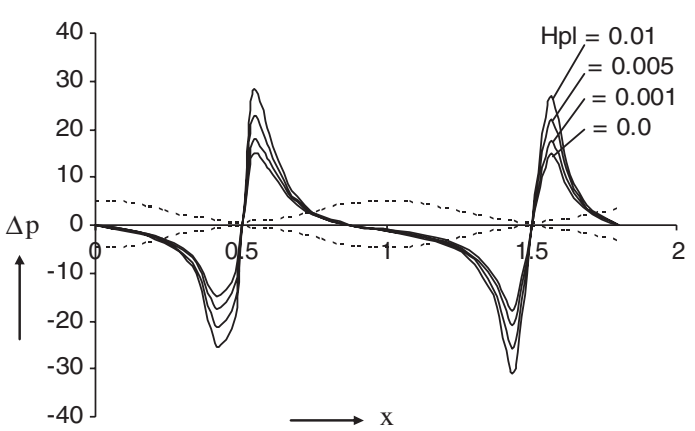

(c) $t=0.5$

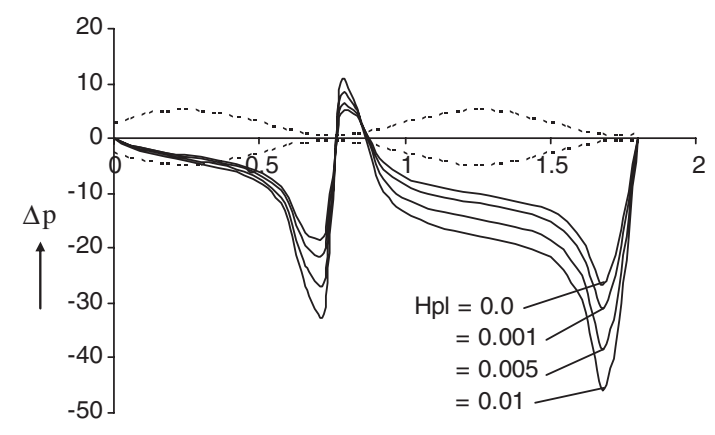

(d) $t=0.75$

Fig. 5. Pressure vs. axial distance. Dotted lines represent the position of bolus and solid lines show the pressure difference distribution, based on Eq. (26), across the length of the tube passing Casson fluid for different radii of plug flow region $\left(H_{p l}=0.0,0.001,0.005,0.01\right), \phi=0.9$ and $l=1.8$.

broadens with increasing non-Newtonian nature of the fluid. Hence, it is concluded that such a fluid is more prone to reflux. This may be physically interpreted as the growing possibility of retrograde motion that takes place near the boundary of the tube.

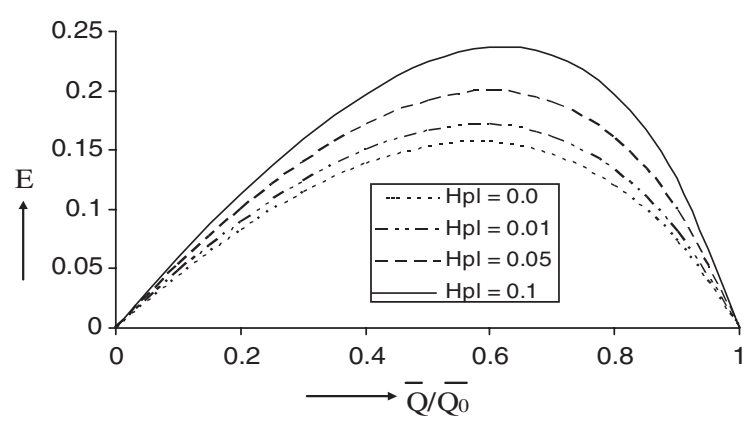

Fig. 6. Mechanical efficiency vs. ratio of average flow rate and maximum averaged flow rate, different lines represent efficiency of peristaltic pump, based on Eq. (36), for corresponding radii of plug flow region $\left(H_{p l}=0.0,0.01,0.05,0.1\right)$ and $\phi=0.4$.

\subsection{Trapping}

A streamline at high flow rates enclosing a bolus of fluid particles is called trapping. Streamlines for Casson fluid with different plug flow regions for $H_{p l}=$ $0.001,0.01,0.1,0.2, \bar{Q}=0.6$ and $\phi=0.6$ are plotted

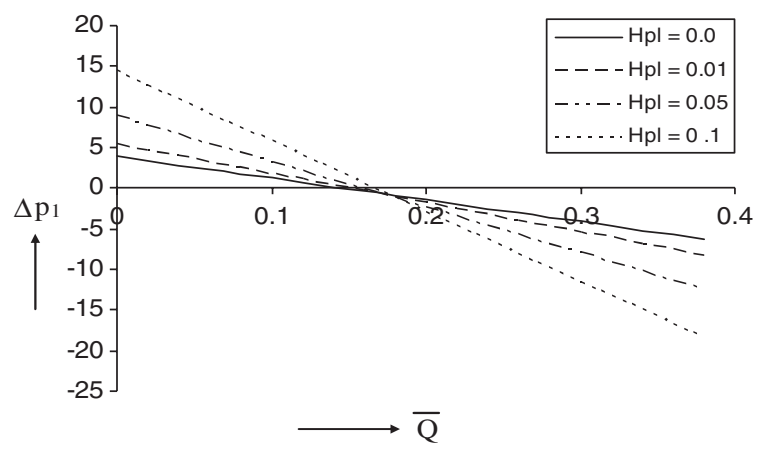

Fig. 7. Pressure across one wavelength vs. averaged flow rate, different lines represent pressure difference, based on Eq. (37), for corresponding radii of plug flow region $\left(H_{p l}=0.0,0.01,0.05,0.1\right)$ $\& \phi=.4$. 


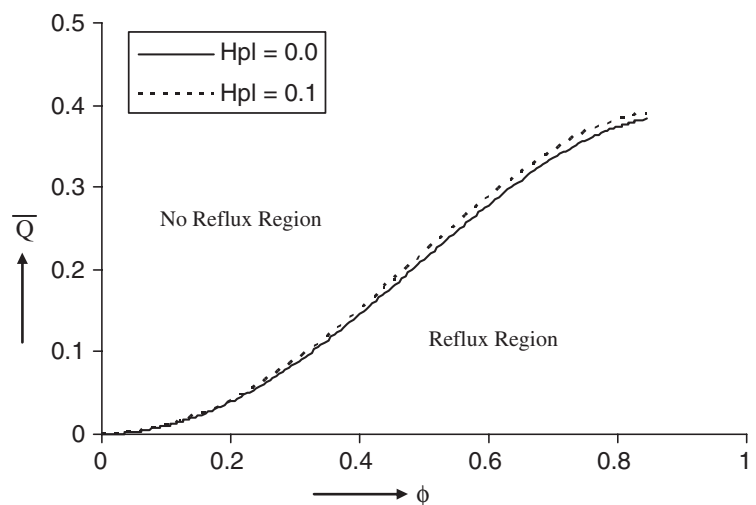

Fig. 8. Time-averaged flow rate vs. amplitude, the different types of line represent reflux limit, based on Eq. (48), for corresponding radii of plug flow region $\left(H_{p l}=0.0,0.1\right)$

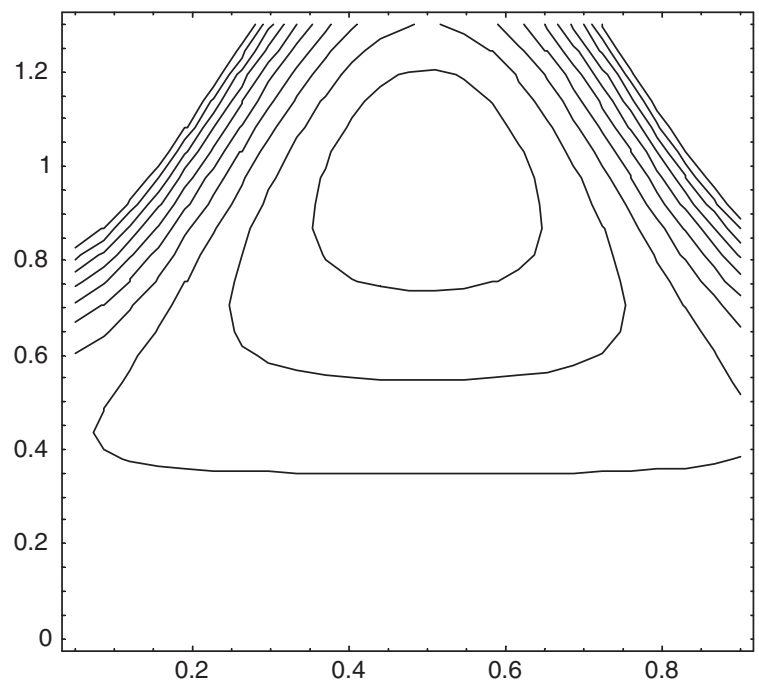

Fig. 9. Streamlines in the wave frame when $\bar{Q}=0.6, \phi=$ $0.6, H_{p l}=0.001$.

in Figs. 9-12. It is revealed that the size of trapped bolus reduces with increase in the plug flow region.

\section{Physical interpretations and concluding remarks}

The two cases of integral and non-integral number of waves in the tube have different patterns of pressure distribution. The former case has an even distribution of pressure whereas that in the latter case is uneven and complex with pressure peaks oscillating in the course of the flow at different instants of time.

Moreover, it is concluded from the examination of plug flow region that the oesophagus has to function more efficiently, i.e., it ought to make additional efforts

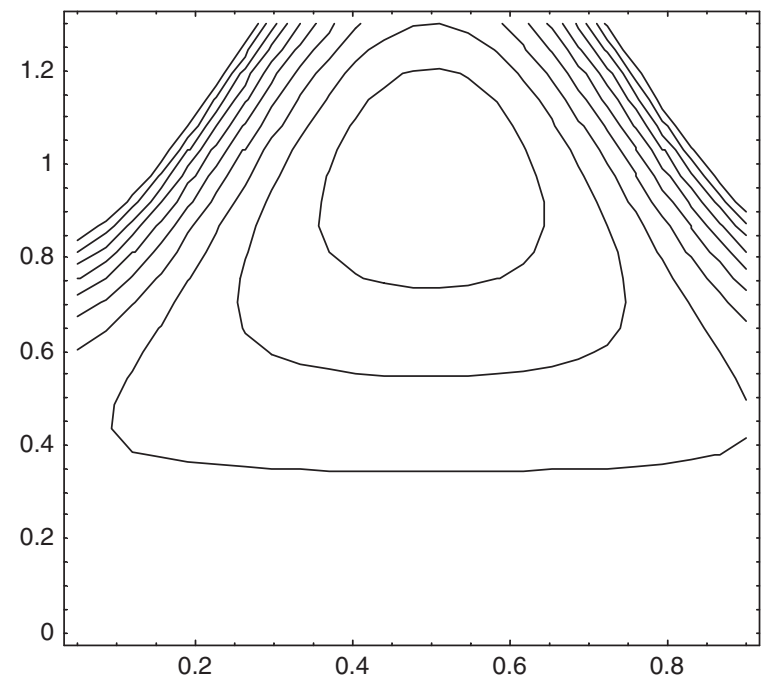

Fig. 10. Streamlines in the wave frame when $\bar{Q}=0.6, \phi=$ $0.6, H_{p l}=0.01$.

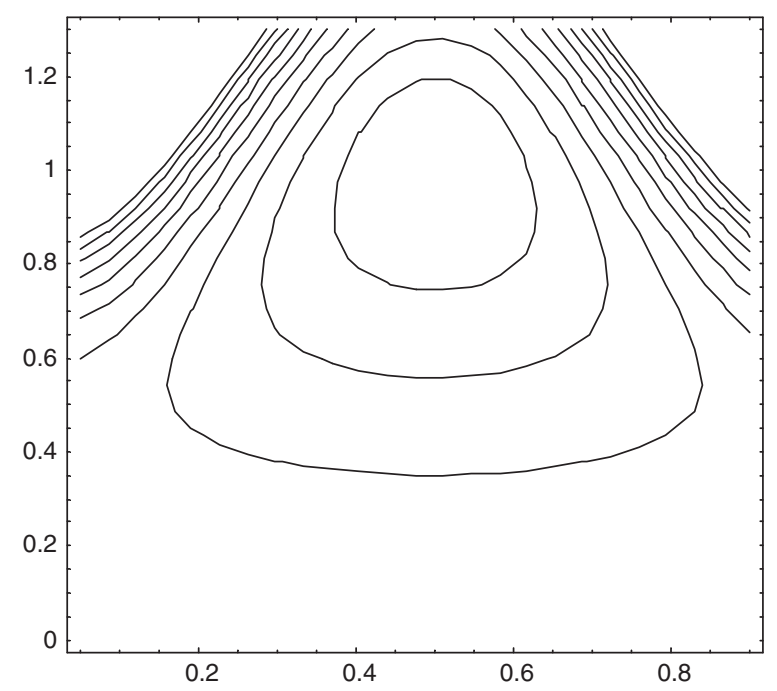

Fig. 11. Streamlines in the wave frame when $\bar{Q}=0.6, \phi=$ $0.6, H_{p l}=0.1$.

to push a Casson fluid down into the stomach. It may be interpreted that concentrated fluids such as jelly, tomato puree, soup, honey and fruits juices etc. cannot be swallowed as easily as Newtonian fluids such as water.

It is further observed that the local wall shear stress increases substantially as the plug flow region widens. This trend is not at all altered by temporal changes. This supports the inference that it rather requires efforts to swallow more concentrated fluids.

For the plug flow region of fixed width, it is found that distribution is similar to the local wall shear stress 


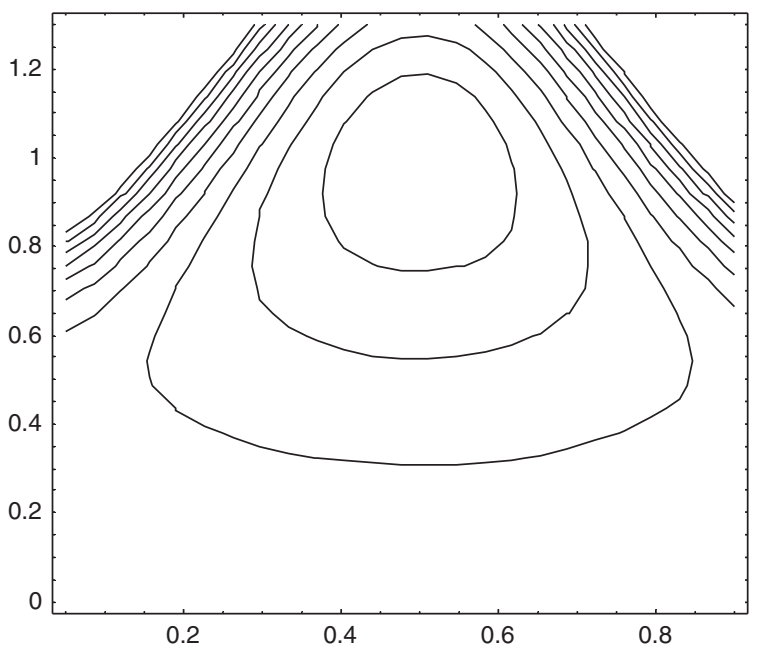

Fig. 12. Streamlines in the wave frame when $\bar{Q}=0.6, \phi=$ $0.6, H_{p l}=0.2$.

distribution. However, quantitatively it is quite small.

As the plug flow region widens, the pressure difference increases, indicating thereby that the averaged flow rate will be less for a Casson fluid, and eventually for concentrated fluids.

The efficiency of an oesophagus from pumping point of view carrying Casson fluid increases with the width of the plug flow region. It is physically interpreted that the oesophagus has to make extra efforts to swallow fluids with higher concentration.

It is observed that the reflux region increases with the increase in the Casson nature. It is inferred from it that such a fluid is more prone to reflux. That is, when someone tries to swallow jelly, tomato puree, soup, honey and fruits juices etc., he has to be more alert as reversal is more probable than when he drinks water.

Finally, it may be concluded that with rising concentration either the number of boluses or the amount of the content will reduce in the oesophagus.

\section{References}

[1] J.C. Misra and S.K. Pandey, Peristaltic transport of blood in small vessels: Study of a mathematical model, Comp Math with applications 43 (2002), 1183-1193.

[2] A.V. Mernone and J.N. Mazumdar, A mathematical study of peristaltic transport of a Casson fluid, Math and Comp Mod 35 (2002), 895-912.

[3] P.N. Rani and G. Sarojamma, Peristaltic transport of a Casson fluid in an asymmetric channel, Australas Phys Eng Sci Med 27 (2004), 49-59.

[4] T. Hayat, N. Ali and S. Asghar, Hall effects on peristaltic flow of a Maxwell fluid in porous medium, Physics Letters A 363 (2007), 397-403.
[5] D. Tsiklauri and Igor Beresnev, Non-Newtonian Effects in the Peristaltic flow of a Maxwell Fluid, Phys Rev E 64 (2003), 036303.

[6] T. Hayat, N. Ali and S. Asghar, Peristaltic motion of a Burger's fluid in a planner channel, Appld Math and Comp 186 (2007), 309-329.

[7] A.R. Rao and M. Mishra, Peristaltic transport of a powerlaw fluid in a porous Tube, J Non-Newtonian Fluid Mech 121 (2004), 163-174.

[8] J.C. Misra and S.K. Pandey, Peristaltic transport of a nonNewtonian fluid with a peripheral layer, Int J Eng Sci 37 (1999), 1841-1858.

[9] J.C. Misra and S.K. Pandey, Peristaltic flow of a multilayered power-law fluid through a cylindrical tube, Int J Eng Sci 39 (2001), 387-402.

[10] V.P. Srivastava and L.M. Srivastava, Influence of wall elasticity and poiseuille flow on peristaltic induced flow of a particle-fluid mixture, Int J Eng Sci 35 (1997), 1359-1386.

[11] T. Hayat, N. Ali and Z. Abbas, Peristaltic flow of a micropolar fluid in channel with different wave forms, Physics Letters A 370 (2007), 331-344

[12] P. Mathu, B.V.R. Kumar and P. Chandra, Peristaltic motion of micropolar fluid in circular cylindrical tubes: Effects of wall properties, Appld Math Mod 32 (2008), 2019-2033.

[13] D. Srinivasacharya, M. Misra, and A.R. Rao, Peristaltic transport of a micropolar fluid in a tube, Acta Mechanics 161 (2003), 165-178.

[14] K. Vajravelu, S. Sreenadh and V.R. Babu, Peristaltic pumping of Herschel-Bulkley fluid in channel, Appld Math and Comp 169 (2005), 726-735.

[15] K. Vajravelu, S. Sreenadh and V.R. Babu, Peristaltic transport of Herschel-Bulkley fluid in an inclined tube, Int J Non-Linear Mech 40 (2005), 83-90.

[16] Meijing Li and J.G. Brasseur, Non-steady peristaltic transport in finite-length Tubes, J Fluid Mech 248 (1983), 129-151.

[17] J.C. Misra and S.K. Pandey, A mathematical model for oesophageal swallowing of a food bolus, Math and Comp Mod 33 (2001), 997-1009.

[18] S.K. Pandey and D. Tripathi, Influence of magnetic field on peristaltic flow of viscous fluid through a finite length cylindrical tube, Applied Bionics and Biomechanics 7 (2010), 169-176.

[19] S.K. Pandey and D. Tripathi, Peristaltic flow characteristics of Maxwell and magneto-hydrodynamic fluids in finite channels: Models for oesophageal swallowing, Journal of Biological Systems 18 (2010), 621-647.

[20] D.H. Pelegrine, F.C. Silva and C.A. Gasparetto, Rheological behavior of pineapple and Mango Pulps, Lebensm-Wiss uTechnol 35 (2002), 645-648.

[21] H.A. Barnes, J.F. Hutton and K. Walters, An introduction to Rhyology, Elsevier, Amsterdam, 1989.

[22] A.H. Shapiro, M.Y. Jafferin and S.L. Weinberg, Peristaltic pumping with long wavelengths at low Reynolds number, $J$ Fluid Mech 35 (1969), 669-675.

[23] W.J. Dodds, Radiology of oesophagus, In Margulis, A.R. Burhenne, H.J. (eds): Alimentary Tract Radiology, 3rd ed (1983), 1529-1603.

[24] J. Ren, B.T. Massey, W.J. Dodds, M.K. Kern, J.G. Brasseur, R. Shaker, S.S. Harrington, W.J. Hogan and R.C. Arndorfer, Determinants of intrabolus pressure during esophageal peristaltic bolus transport, Am J Physiol 264 (1993), G407-G413. 

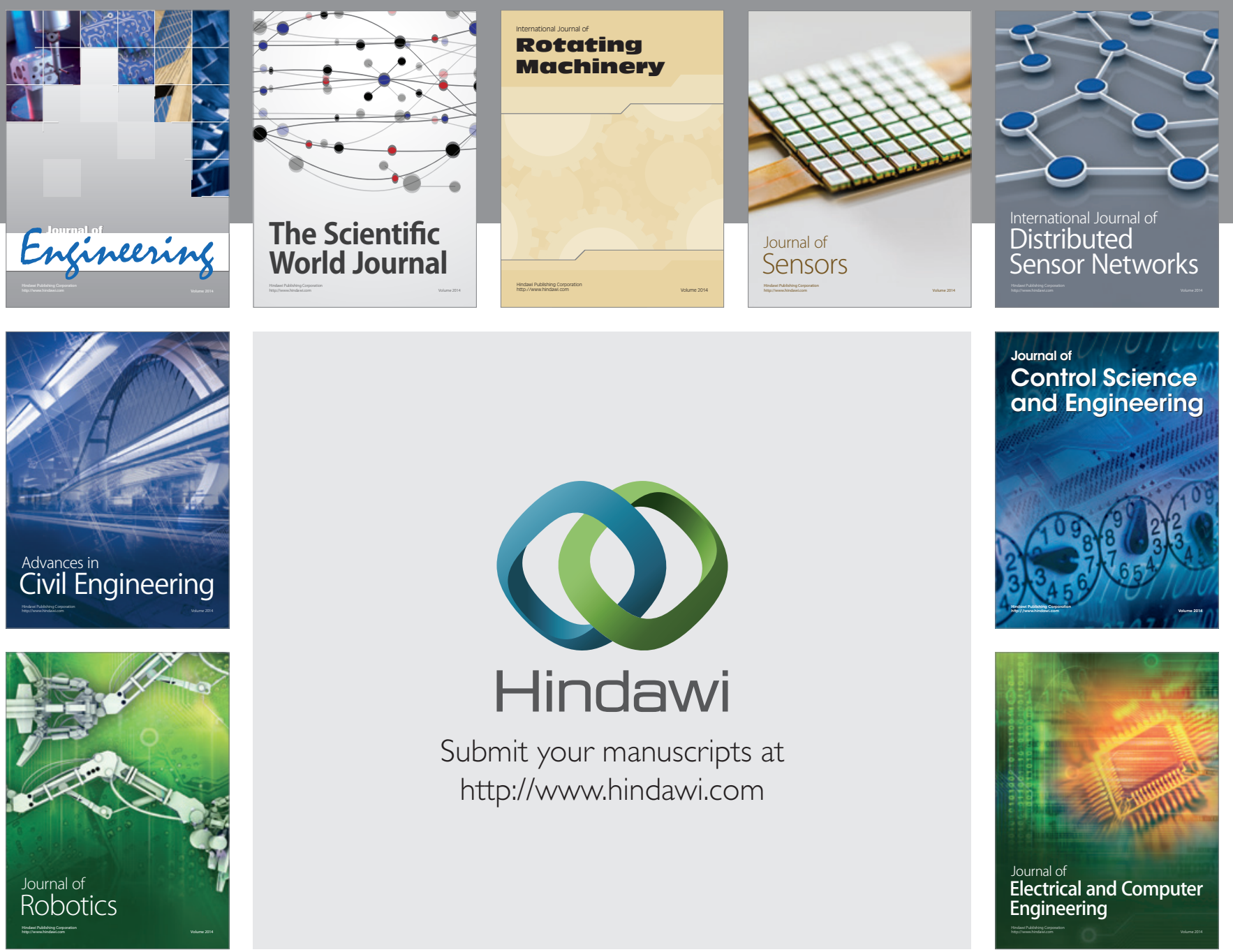

Submit your manuscripts at

http://www.hindawi.com
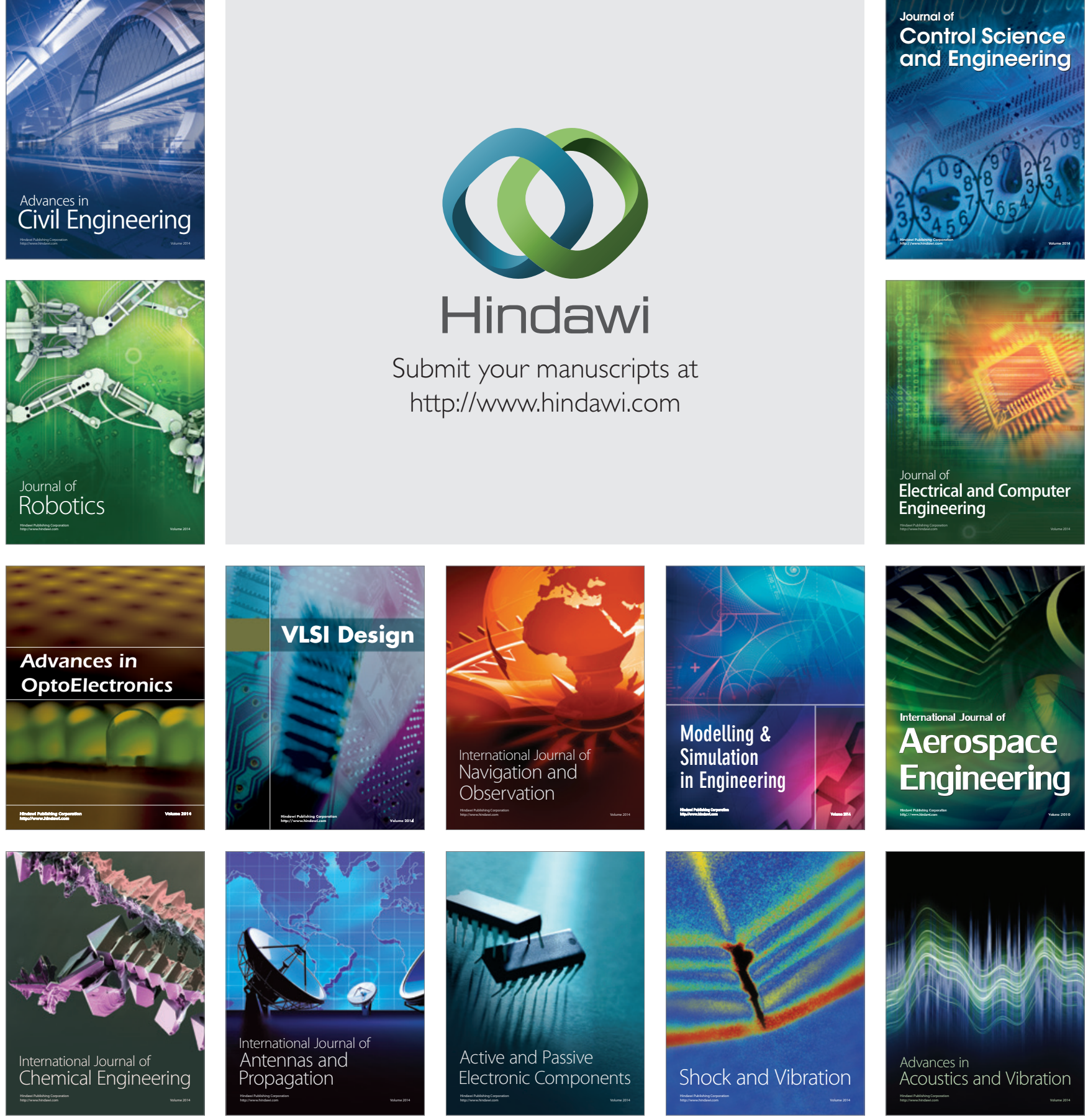